\title{
BMJ Open Access delays to essential surgical care using the Three Delays Framework and Bellwether procedures at Timor Leste's national referral hospital
}

\author{
Dominic Bagguley, ${ }^{\oplus 1}$ Andrew Fordyce, ${ }^{2}$ Jose Guterres, ${ }^{3}$ Alito Soares, ${ }^{3}$ \\ Edgar Valadares, ${ }^{3}$ Glenn Douglas Guest, ${ }^{4}$ David Watters ${ }^{5,6}$
}

To cite: Bagguley D, Fordyce A, Guterres $\mathrm{J}$, et al. Access delays to essential surgical care using the Three Delays Framework and Bellwether procedures at Timor Leste's national referral hospital. BMJ Open 2019;9:e029812. doi:10.1136/ bmjopen-2019-029812

- Prepublication history and additional material for this paper are available online. To view these files, please visit the journal online (http://dx.doi org/10.1136/bmjopen-2019029812).

Received 27 February 2019 Revised 25 June 2019 Accepted 11 July 2019
Check for updates

(C) Author(s) (or their employer(s)) 2019. Re-use permitted under CC BY-NC. No commercial re-use. See rights and permissions. Published by BMJ.

For numbered affiliations see end of article.

Correspondence to Dr Dominic Bagguley; dominic.bagguley@gmail.com

\section{ABSTRACT}

Objectives Our objectives were to characterise the nature and extent of delay times to essential surgical care in a developing nation by measuring the actual stages of delay for patients receiving Bellwether procedures.

Setting The study was conducted at Timor Leste's national referral hospital in Dili, the country's capital.

Participants All patients requiring a Bellwether procedure over a 2-month period were included in the study. Participants whose procedure was undertaken more than 24 hours from initial hospital presentation were excluded.

Primary and secondary outcome measures Data pertaining to the patient journey from onset of symptoms to emergency procedure was collected by interview of patients, their treating surgeons or anaesthetists and the medical records. Timelines were then calculated against the Three Delays Framework.

Results Fifty-six patients were entered into the study. Their mean delay from symptom onset to entering the anaesthesia bay for a procedure was 32.3 hours (+/-11.6). The second delay (4.1+/-2.5 hours) was significantly less than the first $(20.9+/-11.5$ hours; $p<0.005)$ and third delays $(7.2+/-1.2$ hours; $p<0.05)$. Additionally, patients with acute abdominal pain (of which $18 / 20$ ultimately had open appendicectomy and two emergency laparotomies) had a delay time of 53.3 hours (+/-21.3), significantly more than that for emergency caesarean $(22.9+/-18.6$ hours; $p<0.05$ ) or management of an open long-bone fracture (15.5+/-5.56 hours; $p<0.05$ ).

Conclusions Substantial delays were observed for all three stages and each Bellwether procedure. This study methodology could be used to measure access and the three delays to emergency surgical care in low/middleincome countries, although the actual reasons for delay may vary between regions and countries and would require a qualitative study.

\section{INTRODUCTION}

Timor Leste (East Timor), a Southeast Asian nation, has a population of 1.3 million people with an average life expectancy of 68.9 years (global average is 72.0 years). Seventy per cent of the population reside in rural or remote areas. $^{1-3}$ The average gross national income (GNI) per capita in Timor Leste is US\$2290,
Strengths and limitations of this study

- The size of the research project limits the extractability of its results-it was conducted over a short period of time (2 months), in a single hospital, serving a unique population.

- The study was conducted during Timor Leste's dry season. As such, delays associated with roads and infrastructure in a region prone to seasonal flooding were not experienced during the time period of this study.

With respect to the collection of data, open appendicectomy was used as a proxy for patients with acute abdominal pain who might need an emergency laparotomy given the limited number of emergency laparotomies (2) performed during the study period.

- This study's methodology uses the Lancet Commission on Global Surgery's Three Delays Framework for measuring access to essential surgical care in developing nations. It generates actual access delay times rather than estimated 2-hour travel times (relevant only to the second delay).

- While limited to its extractability globally, on a local/ national scale it has highlighted areas for further study and improvement with respect to reducing access delays to essential surgical care in Timor Leste.

making it a low/middle-income country (LMIC) - a per capita GNI between US $\$ 996$ and US\$3895. ' 4 Under the United Nations' Development Program 'Human Development Index', Timor Leste ranks 133 of 188 countries with almost $41.8 \%$ of its population below the national poverty line. ${ }^{56}$

In 2015, the Lancet Commission on Global Surgery (LCoGS) recommended six indicators to enable measurement of health system performance with regard to the provision of access to safe, affordable surgical and anaesthesia care when needed. ${ }^{7}$ One of these key indicators is access to a facility capable of providing three Bellwether procedures ${ }^{8}$ emergency laparotomy, caesarean section 
- The First Delay: The delay in patients seeking care. Reasons for this may be due to financial or geographic restrictions, cultural beliefs, poor health literacy, a history of disconnect from formal health systems, and low awareness of available services or low confidence in those services.

- The Second Delay: The delay in patients reaching care. This can increase when: hospitals are scarce and long distances must be travelled by patients requiring essential surgical care; there are limited private/public transportation options; there is poorly developed public infrastructure; and ambulance services are unable to meet population requirements.

- The Third Delay: The delay in patients receiving surgical care after they have arrived at a hospital. This can be the result of shortages in appropriately skilled staff, medicines and/or infrastructure, such as unreliable electricity, no running water, or inability to provide oxygen.

Figure 1 The Three Delays Framework ${ }^{710}$

and appropriate management of an open fracture-and being able to do so within 2 hours. The Commission report set a target of a minimum of $80 \%$ population coverage by 2030 . In 2017 , only $50 \%$ of Timor Leste's population could access a hospital capable of performing the three Bellwethers within 2 hours. ${ }^{9}$

The Three Delays Framework is another tool recommended by the LCoGS that can be used to quantify the actual stages of delay to emergency surgical care. ${ }^{10}$ This framework breaks down the total delay from symptom onset to the ultimate receipt of surgical care into three distinct stages (see figure 1). Previous studies have based their travel times on geospatial estimates for the second delay, transport time to a Bellwether-capable facility. ${ }^{9} 1112$

This study aimed to use the Three Delays Framework and measure the actual delays at each stage faced by patients receiving Bellwether procedures in Timor Leste, an LMIC.

\section{METHODS}

This prospective study measured the first, second and third delays for patients receiving Bellwether procedures at Dili's Hospital Nacional Guido Valadares (HNGV) over a 2-month period-4 September-4 November 2017. HNGV is Timor Leste's national referral hospital and is one of only two Bellwether-capable hospitals in the country. It comprises 55 surgical and obstetric beds and 3 operating theatres. The surgical, anaesthesia and obstetric workforce comprised 17 surgeons, 5 anaesthetists and 8 obstetricians. There were also eight trained nurse anaesthetists. The hospital manages all emergency and essential conditions for the region and acts as a referral hospital for the other five provincial hospitals in the country, only two of which are Bellwether capable. Some congenital conditions are kept for visiting specialist teams, as there is no specialist paediatric or cardiac surgeon in the country. Since 2001, the Royal Australasian College of Surgeons has managed an Australian Government-funded programme that has supported service delivery and capacity building through specialist surgical and anaesthesia training in Timor Leste. Additionally, a Cuban mission has focused on specialist service delivery and medical graduate training. ${ }^{13}$

The three Bellwether procedures (emergency caesarean, emergency laparotomy and appropriate operative management of open long-bone fracture) were defined by LCoGS. ${ }^{78}$ Our definition of laparotomy included open appendicectomy. Additionally, while the LCoGS specified only open fractures, we included only open long-bone fractures (femur, tibia, humerus and forearm) to avoid including open fractures of the hand and foot.

The data collected on each patient having one of the Bellwether procedures included times of symptom onset, decision to travel, times for travel, distance and mode of travel, arrival time, assessment time and time of arrival in the operating theatre. Patients were identified through admission logs, emergency and surgical ward notes, operating notes and interview (see online supplementary appendix). The patient and/or their relatives, as well as treating specialists, were interviewed within 24 hours to ensure accurate recollection of the relevant times. Any delay attributed to initially presenting to the health system, but to a non-Bellwether equipped facility, was considered part of the second delay. Additionally, for the purposes of our study, we defined 'emergency' as a requirement to receive surgical care within 24 hours of presentation to hospital. Patients whose procedure fell outside of 24 hours from initial presentation to hospital were excluded from the study.

\section{Statistics}

One-way analysis of variance (ANOVA) was performed (Microsoft Excel) to compare times associated with first, second and third delays, as well as the overall time from onset of symptoms to surgery. Post hoc analysis was performed using the Bonferroni procedure.

\section{Patient and public involvement}

Patients were not directly involved in the design of this study, and no patients were involved in either recruitment or the conduct of the study. Nevertheless, senior surgeons, obstetricians and anaesthetists with experience of providing essential surgical care to patients in developing nations, devised the research questions and local protocols. ${ }^{7}$

Due to the practicalities of distributing results within this population and the limited patient involvement in this study, results will not be directly disseminated to participants. Rather, results will be distributed to heads of department at the study site (HNGV, Dili) and the 


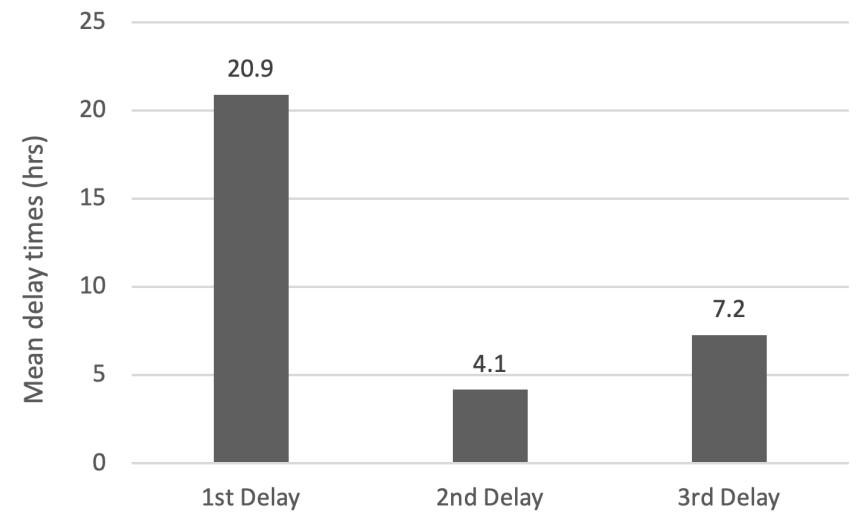

Figure 2 Mean delay times for delay stages (combined Bellwether procedures).

Timor Leste Ministerio Da Saude (Ministry of Health) and the Instituto Nacional De Saude (National Institute of Health).

\section{RESULTS}

A total of 56 Bellwether procedures were recorded within the study period consisting of 20 acute open-abdominal procedures, 25 emergency caesareans and 11 operatively managed open long-bone fractures. Eighteen of the 20 emergency laparotomies were open appendicectomies. The average distance to hospital for 55 of the 56 participants was $8.8 \mathrm{~km}(+/-4.5)$. One patient had travelled from Bali, Indonesia-approximately $1140 \mathrm{~km}$. Of the 56 participants in the study, 30 arrived at hospital via ambulance, a further 21 arrived via private transportation (car/ motorbike) and the remaining 5 by taxi $(n=3)$, walking $(n=1)$ and airplane $(n=1)$. Overall, the total mean delay time from symptom onset to receipt of essential surgical care was 32.3 hours $(+/-11.6)$, irrespective of the Bellwether procedure.

The mean delay time across the Bellwether procedures was greatest for the first delay, followed by the third and second delay stages, respectively (see figure 2). Patients receiving traditional medicine before presentation to a

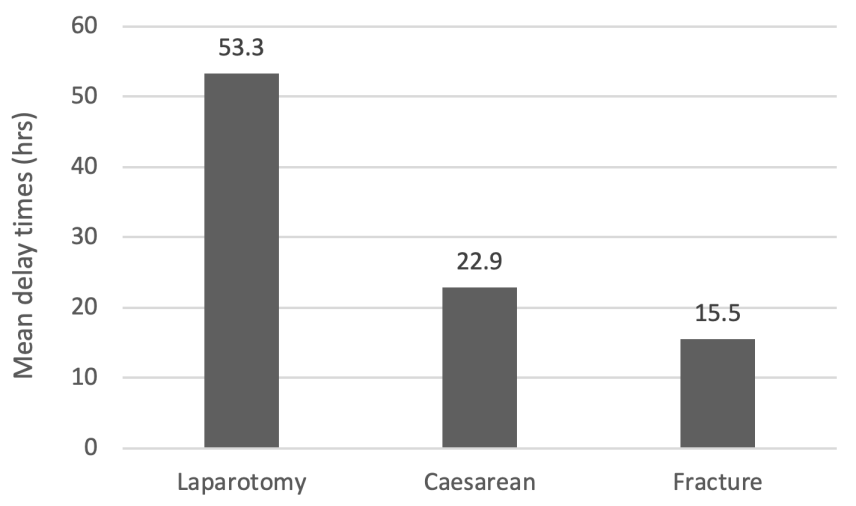

Figure 3 Mean delay times for Bellwether procedures (combined delay stages).

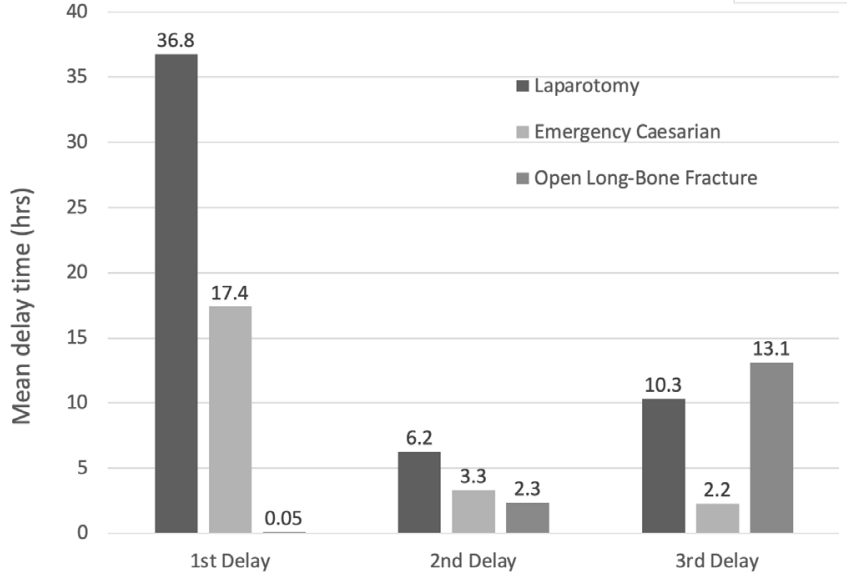

Figure 4 First, second and third mean delay times within and between individual Bellwether procedures.

community health clinic or HNGV was a contributing factor to the first delay in only two cases.

One-way ANOVA demonstrated a significant difference $(p<0.05)$ between the three delay stages, and post hoc analysis determined the mean second delay $(4.1+/-$ 2.5 hours) was significantly less than the first $(20.9+/-$ 11.5 hours; $\mathrm{p}<0.005)$ and third delays $(7.2+/-1.2$ hours; $\mathrm{p}<0.05)$. Patients initially attending community health clinics rather than presenting directly to HNGV $(n=10)$ was a factor that prolonged the second delay stage, however, this was not formally quantified. Thirty-five of the 56 cases $(62.5 \%)$ had a second delay of 2 hours or less.

The mean total delay for individual Bellwether procedures was 53.3 hours (+/-21.3) for emergency abdominal procedures, 22.9 hours $(+/-18.6)$ for emergency caesarean and 15.5 hours $(+/-5.56)$ for management of open longbone fracture (see figure 3). One-way ANOVA demonstrated a significant difference $(p<0.05)$ between these mean total delay times for individual Bellwether procedures. Additionally, post hoc analysis determined that emergency laparotomies had a significantly greater mean total delay time than emergency caesareans $(p<0.05)$ and management of open long-bone fracture $(p<0.05)$.

The greatest delay for an individual Bellwether procedure was the first delay stage for emergency laparotomies (36.8+/-22.3 hours), while the least was the first delay stage for open long-bone fracture repairs $(0.05+/-$ 0.1 hours) (figure 4). A one-way ANOVA demonstrated significant differences for the third mean delay times $(\mathrm{p}<0.001)$ between Bellwether procedures, and post hoc analysis demonstrated the third delay time for emergency caesareans $(2.2+/-1.0$ hours $)$ was significantly less than that for emergency laparotomies $(10.3+/-2.8$ hours; $\mathrm{p}<0.001)$ and management of open long-bone fracture $(13.1+/-5.5$ hours; $\mathrm{p}<0.001)$. Irrespective of the Bellwether procedure that would be required, patients waited an average of 2.75 hours $(+/-0.9)$ to be seen by a surgeon after presenting to the HNGV emergency department. After being assessed by a surgeon, the decision to operate was made in approximately $20 \mathrm{~min}(18.6+/-19.4 \mathrm{~min})$, 
with a subsequent delay to theatre of 4.2 hours $(+/-1.4)$. Factors contributing the third delay included inadequate fasting status $(n=9)$, theatre access $(n=5)$, awaiting surgical review $(n=2)$, awaiting imaging results $(n=1)$ and preoperative blood transfusion $(n=1)$.

\section{DISCUSSION}

The ability to perform the three Bellwether procedures is closely associated with being able to provide all essential obstetric, general, basic, emergency and orthopaedic procedures. ${ }^{8}$ Thus, these three operations are reasonable proxies for the ability to deliver at least most of the procedures listed under emergency and essential surgical care, particularly those which are required urgently. The exceptions are congenital anomalies and obstetric fistula, the so-called 'can do' operations that can await a visiting specialist.

What this study has shown is that in Timor Leste, an LMIC, there is a considerable delay in patients deciding their condition needs hospital treatment when they have acute abdominal pain or are commencing labour. However, there is virtually no delay in their making plans to go to hospital if they suffer an open long-bone fracture. The study also quantified the actual second delay time for patients making the journey to hospital in Timor Leste and described the mode of transport used-where only 21 of 56 patients made the journey by ambulance. Considering the second delay, 35 of the 56 patients $(62.5 \%)$ were able to access essential surgical care within 2 hours. This is greater than the $50 \%$ previously reported for the whole country $^{9}$ and reflects that HNGV is the only Bellwether-capable hospital for the capital city, Dili, with a high proportion of the population living in close vicinity. The study also took place during Timor Leste's dry season when roads were not subject to flooding.

The third delay in having an emergency procedure was not affected by the decision to operate which was usually made within 20 mins, though it took almost 3 hours for a surgical assessment to be performed. It is clear that orthopaedic cases waited the longest once having reached hospital, and this is likely due to such cases being scheduled for daytime hours. The 10 hours delay in having an emergency abdominal surgery after arrival at hospital was largely a result of inadequate fasting status, but may also reflect that the 18 of 20 acute abdominal cases were open appendicectomy. This is a limitation of this study given that appendicectomy delays may not be representative of patients requiring a full laparotomy. For example, in many cases of acute appendicitis, symptoms may escalate slowly. Once arrived in the hospital (the third delay), it is not unreasonable to schedule patients to be operated within 12-24hours in the absence of suspected perforation and if the patient's condition permits. Therefore, the laparotomy data will need to be tested on a larger cohort of patients who actually undergo laparotomy rather than appendicectomy.
Further work is needed to explore the reasons for each delay; this study was focused on the actual times. The reasons are likely to vary from region to region and country to country. Surgical outcomes are better with timely surgery, at least when surgery is needed. This is true for emergency caesarean section ${ }^{14}$ when the mother's pelvic floor is at risk and her life. The life and cerebrum of the neonate is also at risk with complications of labour. This has also been shown for emergency laparotomy, ${ }^{15}$ and a delay of 36 hours to decide to go to hospital and a further 6 hours travelling to hospital means the best part of 2 days has passed. These delays have the potential to compromise outcomes, even though it is recognised that some patients require observation prior to a decision being made - as is often the case with appendicectomy, which accounted for the majority $(18 / 20)$ of emergency abdominal surgeries in the study. Despite the limitation that seasonal variations are not addressed over a 2-month study period, we found that a short focused study achieved local buy-in and delivered results which provided the health system with quantitative data on delay times against which to measure access to emergency surgical care.

The finding that patients with an open fracture are prepared to go straight to hospital suggests that better education as to what to do in the event of acute abdominal pain, or when labour is prolonged or becoming complicated, might be successful in shortening the first delay.

The LCoGS metric on access, though useful, relates to a modelled 2-hour journey time. Without real timelines, the estimates represent a proxy for the real travel time to hospital. By measuring the transport method to hospital, we identified that less than half the patients travelled to hospital by ambulance, which is an important finding for the local health system. The first delay (the time taken to decide to go to hospital) and third delay (accessing assessment, decision making and treatment after arrival) need to be measured by each Bellwether facility locally. Only then can the accessibility of emergency surgical services be understood and quantified. There will be great variation between individual hospitals and regions even within a country. Where laparotomies are relatively uncommon (eg, less than one per week) and the Bellwethers need to be studied, using patients with acute abdominal pain who need surgery including appendicectomy may be a reasonable way to measure system access. This study methodology could be used to identify local issues that affect access and the three delays to emergency surgical care in other LMICs. ${ }^{16}$ A qualitative study involving interview around real patient experiences would help identify issues to address in public education; for example what clinical problems require urgent attention and the importance of making the decision to go to hospital promptly.

\section{Author affiliations}

${ }^{1}$ Department of Surgery, Northern Health, Epping, Victoria, Australia ${ }^{2}$ Department of Surgery, Alfred Health, Melbourne, Victoria, Australia ${ }^{3}$ Department of Surgery, Hospital Nacional Guido Valadares, Dili, Timor-Leste 
${ }^{4}$ Department of Surgery, University Hospital Geelong, Geelong, Victoria, Australia

${ }^{5}$ Royal Australasian College of Surgeons, Geelong, Victoria, Australia

${ }^{6}$ Surgery, Deakin University Faculty of Health, Geelong, Victoria, Australia

Acknowledgements The authors wish to thank all the hospital staff members and the patients that were either directly or indirectly involved in this study.

Contributors DW and GDG developed the research question and study methodology. JG, AS and EV coordinated local ethical approval. DB and AF were responsible for coordinating and undertaking data collection. DB was responsible for data analysis. DB and DW were responsible for writing and editing the manuscript. All authors contributed to the key messages, conclusions and recommendations. DB has full access to all the data in the study and had final responsibility for the decision to submit for publication.

Funding statement The authors have not declared a specific grant for this research from any funding agency in the public, commercial or not-for-profit sectors.

Competing interests None declared.

Patient consent for publication Not required.

Ethics approval Ethics approval was obtained through the Timor Leste Ministerio Da Saude (Ministry of Health) and the Instituto Nacional De Saude (National Institute of Health). As the information collected in the study did not exceed what would reasonably be expected to be documented in patient notes (as part of a routine patient history) no additional consenting process was considered necessary by the ethics committee.

Provenance and peer review Not commissioned; externally peer reviewed. Data availability statement Data are available upon reasonable request.

Open access This is an open access article distributed in accordance with the Creative Commons Attribution Non Commercial (CC BY-NC 4.0) license, which permits others to distribute, remix, adapt, build upon this work non-commercially, and license their derivative works on different terms, provided the original work is properly cited, appropriate credit is given, any changes made indicated, and the use is non-commercial. See: http://creativecommons.org/licenses/by-nc/4.0/.

\section{REFERENCES}

1. World Bank. Timor Leste: the world bank. Available: https://data. worldbank.org/country/timor-leste. [Accessed 27 Oct 2018].
2. World Bank. Life expectancy at birth, total (years): the world bank. Available: https://data.worldbank.org/indicator/SP.DYN.LE00.IN. [Accessed 27 Oct 2018].

3. World Health Organisation. Who south-east Asia region: Timor-Leste statistics summary (2002-Present). In: Global health Observatory data, 2013.

4. World Bank. World bank country and lending groups: the world bank. Available: https://datahelpdesk.worldbank.org/knowledgebase/ articles/906519-world-bank-country-and-lending-groups. [Accessed 28 Oct 2018].

5. United Nations Development Programme. Human development indicators - Timor-Leste: the United nations. Available: http://hdr. undp.org/en/countries/profiles/TLS [Accessed 27 Oct 2018].

6. Asian Development Bank. Poverty in Timor-Leste: the Asian development bank. Available: https://www.adb.org/countries/timorleste/poverty [Accessed 27 Oct 2018].

7. Meara JG, Leather AJM, Hagander L, et al. Global surgery 2030: evidence and solutions for achieving health, welfare, and economic development. The Lancet 2015;386:569-624.

8. O'Neill KM, Greenberg SLM, Cherian M, et al. Bellwether procedures for monitoring and planning essential surgical care in low- and middle-income countries: caesarean delivery, laparotomy, and treatment of open fractures. World J Surg 2016;40:2611-9.

9. Guest GD, McLeod E, Perry WRG, et al. Collecting data for global surgical indicators: a collaborative approach in the Pacific region. BMJ Glob Health 2017;2:E000376.

10. Barnes-Josiah D, Myntti C, Augustin A. The "three delays" as a framework for examining maternal mortality in Haiti. Soc Sci Med 1998:46:981-93.

11. Juran S, Broer PN, Klug SJ, et al. Geospatial mapping of access to timely essential surgery in sub-Saharan Africa. BMJ Glob Health 2018;3.

12. Stewart BT, Tansley G, Gyedu A, et al. Mapping population-level spatial access to essential surgical care in Ghana using availability of bellwether procedures. JAMA Surg 2016;151:e161239.

13. Guest GD, Scott DF, Xavier JP, et al. Surgical capacity building in Timor-Leste: a review of the first 15 years of the Royal Australasian College of Surgeons-led Australian Aid programme. ANZ J Surg 2017;87:436-40.

14. Shaw D, Christilaw J, Munjanja SP. Safe surgery globally by 2030 : the essential role of anesthesia, the view from obstetrics. Anesth Analg 2018;126:1109-11.

15. Royal College of Surgeons England. Emergency surgery: standards for unscheduled care. London: Royal College of Surgeons England Professional Standards and Regulation, 2011.

16. Watters DA, Guest GD, Tangi V, et al. Global surgery system strengthening: it is all about the right metrics. Anesth Analg 2018;126:1329-39. 